\title{
Variación altitudinal de caracteres fenológicos y crecimiento inicial en condiciones controladas entre poblaciones de Nothofagus pumilio provenientes del Centro-Oeste de Chubut, Argentina
}

\author{
Altitudinal variation of phenological characters and initial \\ growth under controlled conditions among Nothofagus pumilio \\ populations from Center-West Chubut, Argentina
}

\author{
Víctor A Mondino ${ }^{\text {a* }}$, Mario J Pastorino ${ }^{\text {b,c }}$, Leonardo A Gallo ${ }^{\text {b }}$

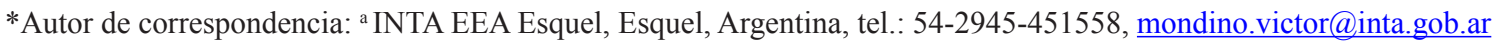 \\ ${ }^{\mathrm{b}}$ INTA EEA Bariloche, Unidad de Genética Ecológica y Mejoramiento Genético Forestal, San Carlos de Bariloche, Argentina. \\ ${ }^{\mathrm{c}}$ CONICET, Argentina.
}

SUMMARY

The genetic differentiation of six natural Argentine populations of lenga (Nothofagus pumilio) from the Center West of the Province of Chubut $\left(42^{\circ} 50 \mathrm{~s}\right.$ ) distributed in an altitudinal range of $500 \mathrm{~m}$ (between 1,000 and 1,500 m a.s.1.) was studied by means of common garden greenhouse trials. Individual growth curves were fitted in one-year-old seedlings using the Boltzmann equation (non-linear regression). Inter-population variation was analyzed with ANOVA in several parameters of the fitted curves, indicative of the increase and the phenology of the growth process. Significant differences were shown between the upper populations and those of the intermediate and lower altitudinal level for the initiation and duration of the growth period, evidencing an ecotypic variation. Our results point out the relevance of considering the altitude of the seed origin in programs of ecological restoration or commercial plantation with lenga.

Key words: adaptive traits, growth curves, genetic variation, lenga.

\section{RESUMEN}

Se estudió la diferenciación altitudinal de seis poblaciones naturales argentinas de lenga (Nothofagus pumilio) del Centro Oeste de la Provincia de Chubut $\left(42^{\circ} 50^{\prime} \mathrm{S}\right)$ distribuidas en un rango altitudinal de $500 \mathrm{~m}$ (1.000 a $1.500 \mathrm{~m}$ s.n.m.) a través de ensayos de ambiente común en invernadero. Se ajustaron curvas individuales de crecimiento en plantines de un año utilizando la ecuación de Boltzmann (regresión no-lineal). Por medio de ANDEVA se analizó la variación inter-poblacional en diversos parámetros de las curvas ajustadas, indicadores del aumento y la fenología del proceso de crecimiento. Se probaron diferencias significativas entre las poblaciones del piso altitudinal superior y las de los pisos altitudinales intermedio y bajo para la iniciación y la duración del periodo de crecimiento, evidenciando un tipo de variación ecotípica. Los resultados prueban la necesidad de considerar la altitud del origen de las semillas en programas de restauración ecológica o plantación comercial con la especie.

Palabras clave: caracteres adaptativos, curvas de crecimiento, variación genética, lenga.

\section{INTRODUCCIÓN}

En árboles de climas templados, la tasa de crecimiento (especialmente en el primer año del establecimiento de las plantas) tiene un fuerte impacto en su sobrevivencia inicial, en las interacciones bióticas que constituye y en su establecimiento a largo plazo (Vitasse et al. 2009). A su vez, la altitud es un factor ambiental que tiene probado efecto en distintas especies forestales sobre la variación genética entre sus poblaciones naturales (Rehfeldt 1986, Premoli 2003). Cambios en la altitud implican cambios ambientales como la temperatura, la presión atmosférica y la concentra- ción de gases, y también cambios indirectos como la humedad atmosférica, la exposición al viento o el desarrollo del suelo (Körner 2007). Estos cambios ambientales son los que pueden conducir a través de procesos de adaptación y de aislamiento reproductivo a la diferenciación genética de poblaciones de pisos altitudinales contrastantes, aun cuando la distancia entre ellas sea escasa. Por ejemplo, con el incremento de la altitud, la duración del período libre de heladas decrece (Körner 2007), y consecuentemente los plantines de poblaciones provenientes de un gradiente altitudinal pueden mostrar adaptación a períodos de crecimiento de diferente longitud (e.g. Vitasse et al. 2009). 
En árboles creciendo bajo condiciones de estrés por bajas temperaturas, una de las características más importantes que contribuyen a su aptitud reproductiva, es la regulación de los eventos fenológicos (i.e. fechas de brotación, de floración y de cierre de yemas). En climas templados, los árboles que brotan tempranamente o tienen un cierre de yemas tardío son más susceptibles a los daños por helada tardía en primavera o temprana en otoño. El daño de los brotes primaverales o de los vástagos aún activos de otoño afecta considerablemente la aptitud de los árboles y reduce el crecimiento anual (Lechowicz 1984). Particularmente en otoño, el daño por helada reduce el almacenamiento de carbono y consecuentemente afecta la tasa de crecimiento del siguiente año (Norby et al. 2003). Esta presión ambiental conduce a que poblaciones naturales creciendo bajo condiciones contrastantes de temperatura sufran un proceso de selección diversificante para fecha de brotación y cierre de yemas (Howe et al. 2003). La adaptación al frío invernal en árboles de climas templados involucra una compleja serie de procesos genéticos, epigenéticos, fisiológicos y de desarrollo (Howe et al. 2003), produciéndose en general un balance entre crecimiento y adaptación al estrés. Así, muchos rasgos fisiológicos que incrementan la resistencia al frío son seleccionados en detrimento del crecimiento (Körner y Paulsen 2004). Estudiar la variación en el ritmo de crecimiento anual de una especie forestal de climas templados permite entonces inferir procesos de adaptación y diferenciación. Conocer estos procesos puede ser crucial para su uso en plantaciones tanto comerciales como de restauración de ecosistemas degradados.

Existen varios estudios de variación inter-poblacional en el ritmo de crecimiento y en apertura y cierre de yemas en especies forestales de zonas templadas, como Pseudotsuga menziesii (Mirb.) Franco (Rehfeldt 1979), Pinus ponderosa Douglas ex C. Lawson (Rehfeldt 1986), Populus sp. (Frewen et al. 2000), Pinus sylvestris L. (Notivol et al. 2007) y Austrocedrus chilensis (D. Don) Pic. Ser. et Bizzarri (Aparicio et al. 2010).

Nothofagus pumilio (Poepp. et Endl.) Krasser (lenga) es una especie forestal nativa de los Bosques Andino-Patagónicos de Chile y Argentina, caducifolia, que ocupa el piso altitudinal más alto de este ecosistema, formando masas boscosas puras. Es un árbol de primera magnitud, con alturas que superan los $35 \mathrm{~m}$ y diámetros de hasta $1,50 \mathrm{~m}$ con una madera de alta calidad para el aserrado, por la que sus bosques son de gran importancia comercial, además de ecológica. A lo largo de su amplia distribución natural (solo en Argentina ocupa más de un millón de hectáreas; Secretaría de Ambiente y Desarrollo Sustentable de la Nación 2005) atraviesa marcados gradientes ambientales, entre ellos el altitudinal. En una misma área la lenga puede ocupar un rango altitudinal de $600 \mathrm{~m}$ de desnivel (Stecconi 2006).

Como antecedente de estudios de variación en caracteres cuantitativos entre poblaciones naturales de altitud contrastante en especies del género Nothofagus, entre los trabajos pioneros está el de Wilcox y Ledgard (1987), quienes a través de ensayos de ambiente común reportaron variación clinal altitudinal en altura de plantines para $N$. menziesii (Hook F.) Oerst. y para N. solandri var. cliffortioides (Hook F.) Oerst., resultando más altos los plantines de los orígenes de menor altitud. Con mediciones en el bosque natural, Donoso (1987) halló en N. obliqua (Mirb.) Oerst. variación clinal en el peso de semilla y el número de estambres en función del gradiente altitudinal, disminuyendo estos parámetros con la altitud.

La variación entre poblaciones naturales de lenga de altitud contrastante ha sido estudiada evaluando diferentes caracteres métricos, tanto morfológicos en plantines (Premoli et al. 2007), como seminales (Cuevas 2000) y fenológicos (Rusch 1993, Barrera et al. 2000, Premoli et al. 2007). Casi todos estos estudios se basan en el análisis de caracteres in situ, o sea sobre los árboles creciendo en forma natural en el bosque, por lo cual no es posible discriminar en qué medida las observaciones se deben a respuestas genéticas o plásticas. Solo el estudio de Premoli et al. (2007) se basa en un ensayo de ambiente común, lo que permitiría hacer inferencias genéticas. Sin embargo, el ensayo fue establecido con plantines transplantados del bosque natural, o sea habiendo ya pasado el primero y más severo proceso de selección que una generación atraviesa a lo largo de su existencia. Solo la producción de plantas bajo condiciones óptimas aseguraría contar en el ensayo con el acervo genético completo de la generación.

En estos estudios se han probado diferencias entre puntos de muestreo en un rango altitudinal en las fechas de brotación y cierre de yemas, y en la duración del período vegetativo (Rusch 1993, Barrera et al. 2000, Premoli et al. 2007). También se han reportado diferencias en el tamaño, cantidad, forma, sanidad y poder germinativo de las semillas en relación con la variación altitudinal (Cuevas 2000).

La hipótesis central del presente trabajo es que la longitud del período anual de crecimiento es un carácter controlado genéticamente y varía con la altitud de origen de la semilla, lo que evidenciaría la ocurrencia de adaptación local en el gradiente altitudinal que ocupa la especie. El objetivo es determinar la posible existencia de un patrón de variación genética en caracteres de crecimiento temprano entre poblaciones naturales de lenga muestreadas a lo largo de un gradiente altitudinal.

\section{MÉTODOS}

De febrero a marzo de 2005 se cosecharon semillas de lenga en dos cordones montañosos contiguos del noroeste de la provincia de Chubut, cerca de Esquel, definiendo así dos áreas de muestreo: Cerro La Hoya del Cordón Esquel y paraje Huemules del Cordón Rivadavia. En cada una de las dos áreas se cosecharon semillas en tres pisos altitudinales contrastantes (a partir de ahora pisos alto, medio y bajo) (cuadro 1), captando de este modo el gradiente altitudinal en el que se desarrolla la especie. Cada combinación de área y piso altitudinal se consideró una población. 
Cuadro 1. Poblaciones de Nothofagus pumilio cosechadas para el ensayo de ambiente común.

Population seed collected of Nothofagus pumilio for the common environment trial.

\begin{tabular}{lccc}
\hline Población & $\begin{array}{c}\text { Latitud } \\
\mathrm{O}\end{array}$ & $\begin{array}{c}\text { Longitud } \\
\mathrm{E}\end{array}$ & $\begin{array}{c}\text { Altitud } \\
\text { m s.n.m. }\end{array}$ \\
\hline LHb (La Hoya bajo) & $42^{\circ} 51^{\prime}$ & $71^{\circ} 16^{\prime}$ & 1.000 \\
LHm (La Hoya medio) & $42^{\circ} 51^{\prime}$ & $71^{\circ} 16^{\prime}$ & 1.250 \\
LHa (La Hoya alto) & $42^{\circ} 50^{\prime}$ & $71^{\circ} 15^{\prime}$ & 1.450 \\
$\mathrm{Hb}$ (Huemules bajo ) & $42^{\circ} 51^{\prime}$ & $71^{\circ} 27^{\prime}$ & 1.000 \\
$\mathrm{Hm}$ (Huemules medio) & $42^{\circ} 50^{\prime}$ & $71^{\circ} 27^{\prime}$ & 1.200 \\
$\mathrm{Ha}$ (Huemules alto) & $42^{\circ} 48^{\prime}$ & $71^{\circ} 29^{\prime}$ & 1.400 \\
\hline
\end{tabular}

El bosque del Cerro La Hoya corresponde al ecotono con la estepa, es fragmentario y se ubica en un ambiente seco $(500 \mathrm{~mm}$ de precipitación media anual aproximadamente), con exposición sur-suroeste, pendiente moderada a fuerte, y suelos someros con alto porcentaje de pedregosidad. El relieve general es quebrado, atravesado por numerosos cañadones con escorrentías estacionales. En la parte inferior la altura de los árboles alcanza unos $15 \mathrm{~m}$, disminuyendo hasta el límite de la timberline, con alturas de menos de $2 \mathrm{~m}$ y características de bosque achaparrado o rastrero.

El bosque del paraje Huemules tiene carácter mésico (700 $\mathrm{mm}$ de precipitación media anual aproximadamente), exposición este y pendiente moderada, con un paisaje general menos quebrado y arroyos permanentes. Los suelos son más profundos, con textura franco arenosa-arenosa, y depósitos de cenizas volcánicas. El bosque es continuo y compacto, cubriendo la ladera desde los 1.000 m s.n.m. hasta los $1.500 \mathrm{~m}$ s.n.m. La altura de los árboles alcanza unos $20 \mathrm{~m}$ en el piso altitudinal inferior, disminuyendo hacia la timberline como fue descrito para el Cerro La Hoya.

En cada piso altitudinal se cosecharon semillas directamente de un mínimo de 15 árboles, con tijeras de altura, guardando una distancia entre ellos de al menos $30 \mathrm{~m}$ para reducir la probabilidad de cosechar individuos emparentados. Todos los ejemplares cosechados presentaban porte arbóreo. Se cosecharon unas 50 semillas por individuo con las que se constituyó un lote combinado para cada piso altitudinal.

El ensayo se llevó a cabo en el vivero del Campo Experimental Agroforestal de INTA situado en Trevelin, provincia de Chubut ( $43^{\circ} 07^{\prime} \mathrm{S}, 71^{\circ} 33^{\prime} \mathrm{O}, 400$ m s.n.m.). En agosto de 2005 se sembraron en bandejas almacigueras las semillas cosechadas, previa estratificación en frío húmedo por un periodo de 60 días. A medida que las semillas iban germinando las plántulas fueron repicadas a tubetes de $250 \mathrm{~cm}^{3}$ en sustrato inerte (turba-arena en proporción 2:1), disponiéndolas en un diseño experimental de bloques completamente aleatorizados, con tres repeticiones y en parcelas lineales de nueve plantas (27 plantas por piso altitudinal de cada área, 162 plantas totales), de tal modo de establecer un ensayo de ambiente común dentro de invernáculo. El tamaño del ensayo se vio condicionado por el número de plantas logradas con el limitado número de semillas que pudieron cosecharse. Las plantas fueron criadas por dos ciclos vegetativos con un sistema de fertirriego siguiendo un protocolo ajustado para la especie. Si bien el tamaño de las macetas elegidas es algo limitado para la expresión libre de la potencialidad de las plantas (Poorter et al. 2012), dado que el estudio se enfoca a caracteres fenológicos y no de tamaño, se consideró suficiente y conveniente utilizar las macetas habituales en la viverización de la especie.

Durante la segunda temporada de crecimiento se midió en forma repetida la altura total de cada individuo desde el cuello hasta la yema terminal del vástago principal, con una precisión de $1 \mathrm{~mm}$. Las mediciones se realizaron a partir del 23 de agosto de 2006 (considerado día 0 en el modelo) cada una o dos semanas durante cinco meses, realizándose una medición ulterior pasados los seis meses de la primera medición, para verificar la finalización del crecimiento. Se registraron las alturas los días $0,14,28$, $42,48,57,71,78,89,96,114,127,142$ y 183 . La fecha de inicio de medición fue fijada por el momento en el que los primeros plantines del ensayo comenzaron a hinchar las yemas (aún cerradas), y la final cuando en el $90 \%$ de los plantines la medición en curso no difirió de la anterior y se había producido el cierre de las yemas vegetativas.

Con los valores de altura medidos se ajustaron por regresión no lineal curvas explicativas del crecimiento individual de cada planta. Para el ajuste de las curvas se utilizó el software Graph Pad Prism 4.03 (Graph Pad Software, San Diego, CA, USA), escogiéndose entre las funciones sigmoidales simétricas más usadas para describir procesos de crecimiento estacional, la de Boltzmann, ya utilizada en otra especie forestal (Aparicio et al. 2012), y cuya ecuación es:

$$
H_{t}=H_{0}+\frac{H f-H o}{1+e^{\left(\frac{T 50-t}{s}\right)}}
$$

Donde: $H_{t}=$ altura de la planta $[\mathrm{mm}]$ al tiempo $\mathrm{t}$ (en días después de la primer medición), $H_{0}[\mathrm{~mm}]=$ asíntota inferior de la curva (altura al inicio de la segunda temporada de crecimiento), $e=$ número de Euler, $H_{f}[\mathrm{~mm}]=$ asíntota superior de la curva (altura al finalizar la segunda temporada de crecimiento), $t_{50}[$ días] = tiempo necesario para llegar a la mitad del período de crecimiento (tiempo medio), y $\mathrm{S}=$ parámetro relacionado con la pendiente de la curva (forma de curva, valores más altos implican curvas más achatadas).

Se obtuvieron, adicionalmente, cuatro variables de cada curva ajustada: iniciación $\left(t_{10}\right)$ y cesación del crecimiento $\left(t_{90}\right)$, los cuales son los días necesarios para alcan- 
zar el $10 \%$ y el $90 \%$ de la altura total respectivamente (Notivol et al. 2007); la duración del crecimiento (D, en días), que fue calculada como la diferencia entre $t_{90} y t_{10}$; y la tasa máxima de crecimiento $\left(T_{\max }\right.$ en $\mathrm{mm} /$ día), que es la derivada primera de la ecuación en el punto de inflexión de la curva $\left(t_{50}\right)$.

Para poner a prueba la significancia de las diferencias entre las dos áreas y entre las altitudes de cada una de ellas en las medias de las variables estimadas con las curvas individuales de crecimiento, se llevaron a cabo análisis de varianza para cada variable. Previamente se verificaron los supuestos de normalidad y homocedasticidad (prueba de Shapiro-Wilk). Se utilizó el paquete lme4 para modelos lineales generalizados (Bates 2015) del programa R 2.11.1 (R version 2.11.1, R Development Core Team 2008), para el siguiente modelo de efectos fijos y aleatorios:

$$
Y_{i j k l}=\mu+A_{i}+P_{j}+A_{i} * P_{j}+B_{k}+S b_{l}+e_{i j k l}
$$

Donde: $Y_{i j k l}=$ ijkl-ésima observación de la variable, $\mu=$ media general, $A_{i}=$ efecto fijo del piso altitudinal, $P_{j}=$ efecto fijo del área, $B_{k}=$ efecto aleatorio del $k$-ésimo bloque, $S b_{l}=$ error de sub parcela y $e_{i j k l}=$ error aleatorio $\operatorname{NID}\left(0, \sigma_{\mathrm{e}}^{2}\right)$.

La hipótesis nula se puso a prueba con estimaciones MCMC utilizando para ello el paquete estadístico rlanguage del mismo programa $\mathrm{R}$.

\section{RESULTADOS}

A pesar de la limitación de ser simétrica alrededor del punto de inflexión, la ecuación de Boltzmann demostró ser flexible, fácil de interpretar, y ajustó los datos con precisión $\left(R^{2}\right.$ promedio $=0,99, R^{2}$ mínimo $\left.=0,84\right)($ figura 1$)$.

Al finalizar la segunda temporada de crecimiento, los plantines de lenga alcanzaron en las condiciones de invernáculo una altura media general de $29,2 \mathrm{~cm}$, con un máximo absoluto de $54 \mathrm{~cm}$ y un mínimo absoluto de $6 \mathrm{~cm}$. Los valores medios por población para todas las variables consideradas pueden observarse en el cuadro 2 , donde también se aprecia que las variables fenológicas presentan menos dispersión que las de forma de curva y de crecimiento propiamente dichas, siendo la variable de mayor dispersión la altura al iniciar la segunda temporada de crecimiento $\left(H_{0}\right)$.

Los modelos ensayados para todas las variables cotejadas fueron estadísticamente significativos, con una probabilidad de $P<0,05$. No se hallaron interacciones entre áreas y pisos altitudinales para ninguna de las variables analizadas. Tampoco se hallaron diferencias significativas entre áreas para ninguna de las variables analizadas. En cambio, pudieron probarse diferencias significativas debido al efecto de la variación altitudinal para las variables $t_{50}(P=0,006), S(P=0,009), t_{10}(P=0,001)$ y Dur $(P=0,009)$. En las cuatro variables la significancia se debió a las diferencias entre el piso altitudinal superior y las otras dos altitudes ensayadas. Así, en ambas áreas ensayadas los plantines del piso superior iniciaron más tarde el crecimiento, tuvieron un tiempo medio mayor, una menor duración de crecimiento y una curva con una forma más empinada que la de los plantines correspondientes a los otros dos pisos altitudinales (figura 2). Las plantas provenientes del piso altitudinal superior fueron en promedio $\mathrm{y}$ significativamente menos vigorosas que las plantas provenientes de los pisos altitudinales inferiores, en ambas poblaciones.

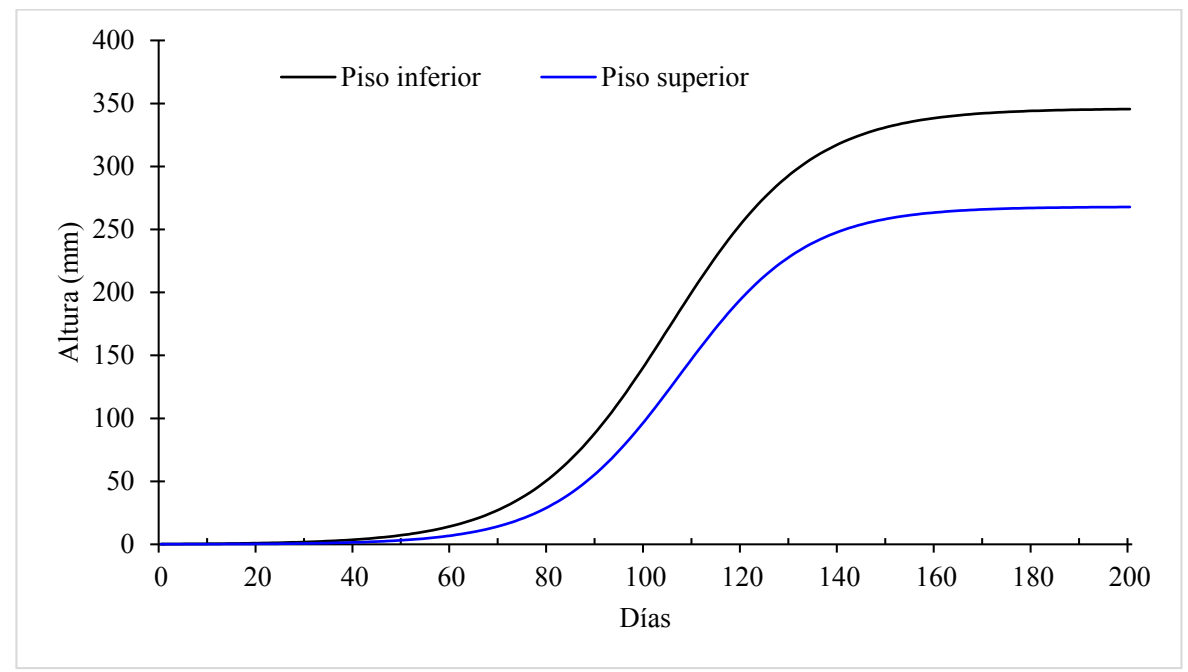

Figura 1. Ejemplos de curvas de crecimiento ajustadas para un individuo originario del piso altitudinal superior y para otro originario del piso altitudinal inferior del Cerro La Hoya.

Examples of adjusted growth curves for an individual originating from the upper altitudinal floor and for another originating from the lower altitudinal floor of Cerro La Hoya. 


\section{DISCUSIÓN Y CONCLUSIONES}

La limitación en el número de semillas producidas y cosechadas, y de plantas logradas en el vivero, impidió llevar a cabo un pre-muestreo para determinar el tamaño óptimo de las parcelas. Sin embargo los valores de coe- ficientes de variación logrados con las parcelas de nueve plantas fueron menores al $20 \%$ en cinco de las ocho variables consideradas, reflejando una dispersión de los datos aceptables para ensayos biológicos de este tipo. Los bajos niveles de variación detectados para los caracteres fenológicos respaldan los tamaños muestrales utilizados en el

Cuadro 2. Medias (CV \%) por población para las variables obtenidas a través del modelo de crecimiento ajustado para cada plantín: $\mathrm{H} 0=$ altura inicial, $\mathrm{Hf}=$ altura final, $\mathrm{t} 50=$ tiempo medio, $\mathrm{S}=$ forma de la curva, Tmáx = tasa máxima de crecimiento, $\mathrm{t} 10=$ inicio de crecimiento, $\mathrm{t} 90$ = cesación de crecimiento, Dur = duración del crecimiento.

Averages $(\mathrm{CV} \%)$ by population for the variables obtained through the adjusted growth model for each seedling: H0 = initial height, $\mathrm{Hf}=$ final height, $\mathrm{t} 50=$ average time, $\mathrm{S}=$ shape of the curve, Tmax $=$ maximum growth rate, $\mathrm{t} 10=\mathrm{start}$ of growth, $\mathrm{t} 90$ $=$ cessation of growth, Dur $=$ duration of growth .

\begin{tabular}{lcccccccc}
\hline Población & $\begin{array}{c}\mathrm{H}_{0} \\
{[\mathrm{~mm}]}\end{array}$ & $\begin{array}{c}\mathrm{H}_{\mathrm{f}} \\
{[\mathrm{mm}]}\end{array}$ & $\begin{array}{c}\mathrm{t}_{50} \\
{[\text { días }]}\end{array}$ & $\mathrm{S}$ & $\begin{array}{c}\mathrm{T}_{\max } \\
{[\mathrm{mm} / \mathrm{d}]}\end{array}$ & $\begin{array}{c}\mathrm{t}_{10} \\
{[\text { días] }}\end{array}$ & $\begin{array}{c}\mathrm{t}_{90} \\
{[\text { días] }}\end{array}$ & $\begin{array}{c}\text { Dur } \\
\text { [días] }\end{array}$ \\
\hline LHb & $65(58)$ & $346(27)$ & $105(6)$ & $14,4(15)$ & $4,9(26)$ & $74(9)$ & $137(7)$ & $63(15)$ \\
LHm & $35(36)$ & $335(26)$ & $101(10)$ & $15,4(19)$ & $4,9(25)$ & $67(13)$ & $135(10)$ & $67(19)$ \\
LHa & $46(62)$ & $268(39)$ & $107(10)$ & $13,0(23)$ & $4,4(43)$ & $78(14)$ & $136(10)$ & $57(23)$ \\
$\mathrm{Hb}$ & $43(56)$ & $316(24)$ & $101(8)$ & $16,2(21)$ & $4,4(31)$ & $65(14)$ & $136(10)$ & $71(21)$ \\
$\mathrm{Hm}$ & $41(41)$ & $320(34)$ & $101(9)$ & $14,6(15)$ & $4,9(42)$ & $69(18)$ & $133(7)$ & $64(15)$ \\
$\mathrm{Ha}$ & $37(33)$ & $290(27)$ & $107(6)$ & $12,8(12)$ & $4,9(27)$ & $79(10)$ & $135(4)$ & $56(12)$ \\
\hline
\end{tabular}

A

B
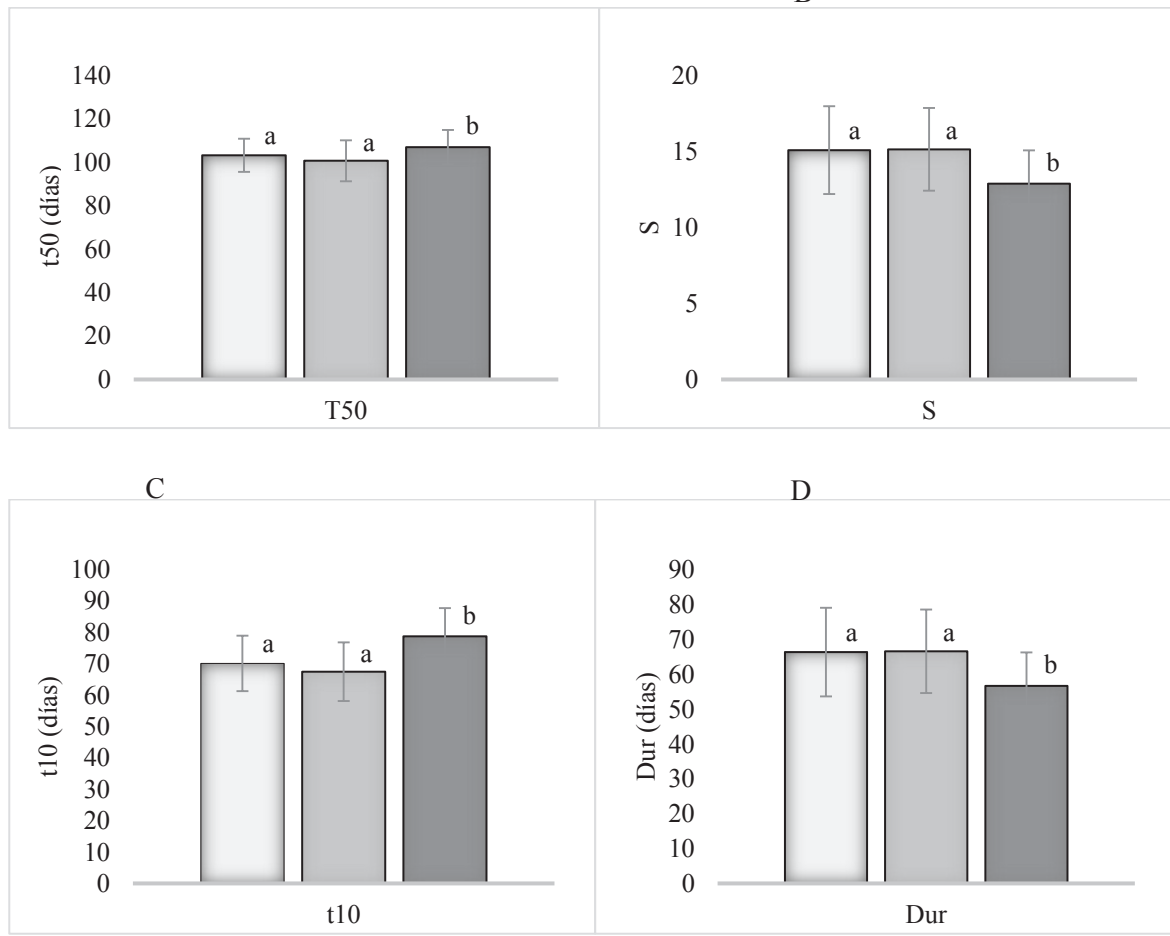

Figura 2. Efecto de la altitud de origen para las variables: A) tiempo medio $\left(T_{50}\right)$, B) forma $(S)$, C) tiempo de inicio de crecimiento $\left(t_{10}\right)$ y D) duración del período de crecimiento $(D u r)$. Letras diferentes indican diferencias significativas con una $P<0,05$. Pisos altitudinales: bajo: medio: alto:

Effect of altitude of origin for the variables: A) mean time (T50), B) form (S), C) time of onset of growth (t10) and D) duration of the growth period (Dur). Different letters indicate significant differences with a $P<0.05$. Altitudinal floors: low: medium: high: 
presente estudio. Sin embargo, la alta variación encontrada en caracteres relacionados al crecimiento en altura $(\mathrm{H} 0$, Hf, Tmáx), evidencian la necesidad de aumentar el tamaño muestral en estudios posteriores sobre la variación genética y fenotípica en lenga.

En este ensayo de ambiente común en vivero, las poblaciones de elevaciones bajas y medias expresaron un mayor potencial de crecimiento a temprana edad, creciendo durante un período de tiempo relativamente más largo. A mayor altitud, las poblaciones mostraron evidencias de una posible adaptación a períodos de crecimiento más cortos, con un inicio de crecimiento más tardío y un crecimiento relativamente más abrupto. Si bien no pudieron probarse diferencias significativas entre los pisos altitudinales en la altura final de los plantines, los menores valores medios medidos corresponden en ambas áreas al piso altitudinal superior, lo que concuerda con el resultado encontrado en las variables con diferencias significativas. Estos resultados confirman la conclusión del trabajo de Premoli et al. (2007) en el que se probó variación en caracteres fenológicos entre rodales de dos pisos altitudinales $(1.100 \mathrm{y}$ $1.540 \mathrm{~m}$ s.n.m.) por medio de ensayos de ambiente común instalados con plantines transplantados desde un bosque natural a vivero. El retraso en el inicio de crecimiento de los árboles de los pisos altitudinales superiores fue probado en ambos estudios de ambiente común realizados en forma independiente, tanto con plantines instalados naturalmente en el bosque como con plantines originados de semillas germinadas en condiciones de vivero.

Las poblaciones de mayores altitudes expresan un bajo crecimiento potencial debido a que los eventos de desarrollo deben ser completados en un corto período libre de heladas intensas. Esta característica también se manifiesta, con distinta intensidad, en otras especies de árboles, tales como Pseudotsuga menziesii (Rehfeldt 1979), Larix occidentalis Nutt. (Rehfeldt 1982), Pinus contorta Douglas. (Rehfeldt 1984) у Pinus ponderosa (Rehfeldt 1986). Este comportamiento coincide con modelos presentados para algunas especies del hemisferio norte (Howe et al. 2003, Aitken 2004). En general el cese precoz de crecimiento se asocia con el incremento de la resistencia a las heladas, ya que los genotipos más resistentes al daño por heladas tienden a tener un cese de crecimiento temprano y un menor periodo de crecimiento (Howe et al. 2003).

La mayoría de los daños provocados por heladas ocurren en la época de brotación, sobre los brotes, o en la época de la cesación del crecimiento, antes de la formación de las yemas de latencia invernal (Timmis et al. 1994). Modelos de daños por heladas sugieren que las heladas de primavera representan el mayor riesgo en la mayoría de las localidades (Timmis et al. 1994). En el presente estudio se encontraron diferencias entre los pisos altitudinales en el momento de brotación (no de fin del crecimiento). Si bien no se ha realizado en el presente trabajo un estudio referido al daño por heladas asociado al gradiente altitudinal, la brotación tardía de las poblaciones de mayor altitud ten- dría un carácter adaptativo que además de prevenir el daño por heladas tardías, tendría como efecto secundario un período de crecimiento más corto. De este modo, se produciría un compromiso entre el incremento del crecimiento producto del adelantamiento de la brotación y el potencial daño por frío producto de heladas tardías. Este efecto fue registrado en otro Nothofagus sp. de Sudamérica. Aparicio et al. (2015) encontraron en un ensayo de orígenes de $N$. obliqua que la población de mayor altitud fue la menos afectada por una helada tardía de comienzos de verano y a la vez la de menor crecimiento en altura.

Analizando in situ la variación en fenología de árboles adultos de lenga en el gradiente altitudinal, Rusch (1993) halló que la brotación foliar es simultánea con la floración, por lo cual, al producirse un retraso en la brotación con el incremento de altitud, también se produce un retraso en la floración y la polinización, y posteriormente en la formación y el llenado de fruto. Este fenómeno conduce al aislamiento reproductivo entre los rodales de la misma especie ubicados en distintos pisos altitudinales, aun cuando la distancia entre los mismos sea menor a la distancia del vuelo del polen efectivo. El aislamiento reproductivo es una de las condiciones necesarias para que las poblaciones evolucionen hacia la diferenciación.

La diferenciación entre las poblaciones fue probada en el material proveniente del piso altitudinal superior respecto a los otros dos pisos altitudinales que se mostraron indiferenciados entre sí, por lo que la variación no sería del tipo clinal, sino ecotípica. Esto no pudo ponerse a prueba en el estudio de Premoli et al. (2007) con variables morfológicas y fenológicas en plantines transplantados, ya que no consideró en el muestreo un piso altitudinal intermedio. En cambio, en el trabajo de Premoli (2003) con marcadores genéticos neutrales (isoenzimas) se incluyeron en el muestreo cuatro pisos altitudinales, por lo que el material fue el apropiado para poner a prueba la hipótesis de variación clinal. En este caso también se probaron diferencias altitudinales, reportando mayor variación genética en las poblaciones del piso altitudinal inferior en contraste con las del piso altitudinal superior, y adicionalmente leves evidencias de variación clinal en el gradiente altitudinal. Sin embargo, el patrón clinal observado es muy leve y solo restringido a algunos de los parámetros genéticos y a algunas de las cuatro áreas de muestreo. Por ejemplo, el nivel de polimorfismo en el Cerro Otto fue idéntico en los tres pisos altitudinales inferiores, $y$ en el Cerro Challhuaco fueron idénticos en los pisos altitudinales inferior e intermedio alto. En cualquier caso, el patrón de variación en caracteres adaptativos no necesariamente debe guardar relación con el patrón en marcadores neutrales, ya que estos no son susceptibles de ser seleccionados por el ambiente.

El proceso de adaptación que habría conducido a la diferenciación encontrada estaría conducido por la duración del fotoperiodo, rasgo a su vez ligado a la probabilidad de ocurrencia de heladas. De este modo el mecanismo de resistencia gana en efectividad ya que logra anticiparse al 
evento de riesgo, o sea, la helada tardía (por la lentitud del proceso, una respuesta fenológica no podría dispararse con el evento en ciernes). Así, las poblaciones del piso altitudinal superior requieren un fotoperiodo más prolongado para desencadenar la fenofase de brotación, ya que de hacerlo con el mismo fotoperiodo de las bajas altitudes los nuevos tejidos correrían un alto riesgo de daño por heladas tardías. El carácter ecotípico de esta variación es evidencia de una respuesta binaria delimitada por un cierto umbral de fotoperiodo a partir del cual las plantas son afectadas, lo cual concuerda con el hecho de que las plantas sufren daño por heladas a partir de una cierta intensidad de frío que sobrepasa su capacidad (estructural y fisiológica) de tolerancia, provocando el colapso de los tejidos (Alberdi et al. 1985). Una probabilidad alta de que ocurra un suceso natural con cierta recurrencia, al considerar tiempos evolutivos se transforma en certeza, de tal modo que en lapsos generacionales un determinado fotoperiodo termina siendo equivalente a una determinada intensidad de frío. El fotoperiodo al que se produce la intensidad de frío que la lenga no es capaz de tolerar en la fenofase de brotación varía según la altitud: a elevadas altitudes se requiere un fotoperiodo más largo para asegurar que no se producirán las heladas de riesgo.

La diferenciación encontrada entre las poblaciones de mayor altitud y las de altitudes media y baja se repite en ambas áreas de estudio, aun cuando representan condiciones diferenciales de humedad. Esto le otorga mayor robustez a las conclusiones.

Los resultados del presente estudio tienen consecuencias relevantes para la silvicultura de implantación de la especie, tanto con fines comerciales como de restauración ecológica, ya que muestran que es necesario considerar la altitud del origen de las semillas que se utilizarán para forestación o reforestación. Restaurar sitios altos con semilla de sitios bajos podría ocasionar daños y alta mortalidad de los plantines por heladas tardías. Análogamente, utilizar semillas de orígenes altos en sitios bajos posiblemente otorgaría desventajas competitivas a los plantines. Más aún: si el azar dispusiera una sucesión de años benéficos sin heladas tardías y los árboles plantados lograran superar la etapa de plantín, y llegando a la adultez introgredieran sus genes en el acervo genético local, el carácter perjudicial del acervo genético exótico podría terminar afectando de manera irreversible a los bosques naturales circundantes.

Por último, parece recomendable continuar este tipo de trabajos estudiando la variación genética de la lenga en los mismos caracteres pero a nivel intra-poblacional, con el fin de analizar la capacidad adaptativa de la especie, lo cual cada vez cobra mayor importancia a la luz del cambio climático global en ciernes.

\section{REFERENCIAS}

Aitken SN. 2004. Genecology and adaptation of forest trees. In Evans J, J Burley, J Youngquist eds. Encyclopedia of Forest Sciences. Amsterdam, The Netherlands. Elsevier. p. 197-204.
Alberdi M, M Romero, D Ríos, H Wenzel. 1985. Altitudinal gradients of seasonal frost resistance in Nothofagus communities of southern Chile. Acta oecologica. Oecologia Plantarum 6: 21-30.

Aparicio AG, MJ Pastorino, LA Gallo. 2010. Genetic variation of early height growth traits at the xeric limits of Austrocedrus chilensis (Cupressaceae). Austral Ecology 35: 825-836. DOI: $\underline{10.1111 / j .1442-9993.2009 .02090 . x}$

Aparicio AG, SM Zuki, MM Azpilicueta, FA Barbero, MJ Pastorino. 2015. Genetic versus environmental contributions to variation in seedling resprouting in Nothofagus obliqua. Tree Genetics \& Genomes 11:23. DOI: 10.1007/s11295$\underline{015-0847-0}$

Barrera MD, JL Frangi, LL Richter. 2000. Structural and functional changes in Nothofagus pumilio forests along an altitudinal gradient in Tierra del Fuego, Argentina. Journal of Vegetation Science 11: 179-188. DOI: 10.2307/3236797

Bates D, M Maechler, B Bolker, S Walker. 2015. Fitting Linear Mixed-Effects Models Using lme4. Journal of Statistical Software 67(1): 1-48. DOI: 10.18637/jss.v067.i01

Cannell MGR, RI Smith. 1984. Spring frost damage of young Picea sitchensis. 2. Predicted dates of budburst and probability of frost damage. Forestry 27: 177-197. DOI: 10.1093/ forestry/57.2.177

Cuevas JA. 2000. Tree recruitment at the Nothofagus pumilio alpine timberline in Tierra del Fuego, Chile. Ecology 88: 840-855. DOI: 10.1046/j.1365-2745.2000.00497.x

Donoso C. 1987. Variación natural en especies de Nothofagus en Chile. Bosque 8(2): 85-97.

Frewen BE, TH Chen, GT Howe, J Davies, A Rohde, W Boerjan, HD Jr Bradsahw. 2000. Quantitative trait loci and candidate gene mapping of bud set and bud flush in Populus. Genetics 154: 837-845.

Howe G, S Aitken, D Neale, K Jermstad, N Wheeler, T Chen. 2003. From genotype to phenotype: unraveling the complexities of cold adaptation in forest trees. Canadian Journal of Botany 81: 1247-1266.

Körner C. 2007. The use of 'altitude' in ecological research. Trends in Ecology and Evolution 22: 569-574. DOI: $\underline{10.1016 / \text { j.tree.2007.09.006 }}$

Körner C, J Paulsen. 2004. A world-wide study of high altitude treeline temperatures. Journal of Biogeography 31: 713732. DOI: $10.1111 / \mathrm{j} .1365-2699.2003 .01043 . \mathrm{x}$

Lechowicz MJ. 1984. Why do temperate deciduous trees leaf out at different times? Adaptation and ecology of forest communities. The American Naturalist 124: 821-842. DOI: $\underline{10.1086 / 284319}$

Norby RJ, JS Hartz-Rubin, MJ Verbrugge. 2003. Phenological responses in maple to experimental atmospheric warming and $\mathrm{CO}_{2}$ enrichment. Global Change Biology 9: 17921801. DOI: $10.1111 /$ j.1365-2486.2003.00714.x

Notivol E, MR García-Gil, R Alía, O Savolainen. 2007. Genetic variation of growth rhythm traits in the limits of a latitudinal cline of Scots pine. Canadian Journal of Forest Research 37: 540-551. DOI: 10.1139/X06-243

Poorter H, J Bühler, D van Dusschoten, JM Climent, JA Postma. 2012. Pot size matters: a meta-analysis of the effects of rooting volume on plant growth. Functional Plant Biology 39: 839._DOI: $10.1071 / \mathrm{FP} 12049$

Premoli AC. 2003. Isozyme polymorphisms provide evidence of clinal variation with elevation in Nothofagus pumilio. Here- 
dity 94: 218-226. DOI: 10.1093/jhered/esg052

Premoli AC, E Raffaele, P Mathiasen. 2007. Morphological and phenological differences in Nothofagus pumilio from contrasting elevations: Evidence from a common garden. Austral Ecology 32: 515-523. DOI: 10.1111/j.14429993.2007.01720.x

R Development Core Team. 2008. R: A language and environment for statistical computing. R Foundation for Statistical Computing, Vienna ISBN 3-900051-07-0.

Rehfeldt GE. 1979. Variation in cold hardiness among populations of Pseudotsuga menziesii var. glauca. Research Paper Int-233. Ogden, UT, USA. USDA Forest Service, Intermountain Forest and Range Experiment Station. 11 p. DOI: $\underline{10.5962 / \text { bhl.title. } 69082}$

Rehfeldt GE. 1982. Diferentation of Larix occidentalis from de northem Rocky Mountains. Silvae Genetica 31: 13-19.

Rehfeldt GE. 1984. Microevolution of conifers in the northern Rocky Mountains: a view from common gardens. In Lanner RN ed. Proceedings of the 8th North American Forest Biology Workshop Symposium on Historical and Genetic Components of Geographic Variation Patterns. Logan, Utah, USA. 30 July - 1 August 1984. p. 132-146.

Rehfeldt GE. 1986. Adaptive Variation in Pinus ponderosa from Intermountain Regions. I. Snake and Salmon River Basin.
Forest Science 32: 79-92.

Rusch V. 1993. Altitudinal variation in the phenology of Nothofagus pumilio in Argentina. Revista Chilena de Historia Natural 66: 131-141.

SAyDS (Secretaría de Ambiente y Desarrollo Sustentable de la Nación). 2005. Primer Inventario Nacional de Bosques Nativos. Proyecto Bosques Nativos y Áreas Protegidas BIRF 4085-AR 1998-2005. p 116.

Stecconi M. 2006. Variabilidad arquitectural de especies nativas de Nothofagus de la Patagonia (N. antarctica, N. pumilio, N. dombeyi). Tesis Doctoral. San Carlos de Bariloche, Argentina. Universidad Nacional del Comahue. $162 \mathrm{p}$.

Timmis R, J Flewelling, C Talbert. 1994. Frost injury prediction model for Douglas-fir seedlings in the Pacific Northwest. Tree Physiology 14: 855-869. DOI: 10.1093/treephys/14.7-8-9.855

Vitasse Y, S Delzon, CC Bresson, R Michalet, A Kremer. 2009. Altitudinal differentiation in growth and phenology among populations of temperate-zone tree species growing in a common garden. Canadian Journal of Forest Research 39: 1259-1269. DOI: 10.1139/X09-054

Wilcox M, J Ledgard. 1987. Provenance variation in the New Zealand species of Nothofagus. New Zealand Journal of Ecology 6: 19-31. 